Pacific Journal of Mathematics

TTRS OF PURTT Y I A AELLAN GROUI 


\section{CENTERS OF PURITY IN ABELIAN GROUPS}

\section{R. S. PierCe}

This note is a supplement to the paper $[5]^{1}$ of J. D. Reid "On subgroups of an abelian group maximal disjoint from a given subgroup." Our main result is based on the observation that in the case of primary groups, a bit of extra information can be gleaned from Reid's Theorem 2.1. We are led to the following characterization of the "centers of purity" in a $p$-group.

Theorem 1. Let $G$ be a p-group. For each integer $k \geqq 0$, define $P_{k}=G[p] \cap p^{k} G$. Let $P_{\infty}=G[p] \cap G^{1}$, and $P_{\infty+1}=P_{\infty+2}=0$. Let $H$ be a subgroup of $G$. Then $H$ is a center of purity in $G$ (that is, every subgroup of $G$ which is maximal with respect to disjointness from $H$ is pure) if and only if there exists $k$ with $0 \leqq k \leqq \infty$ such that

$$
P_{k} \supseteqq H[p] \supseteqq P_{k+2} \text {. }
$$

It is easy to see that if $G$ is a torsion group and $H$ is a subgroup of $G$, then $H$ is a center of purity in $G$ if and only if every $p$-component $H_{p}$ of $H$ is a center of purity in the corresponding $p$ component $G_{p}$ of $G$. Thus, Theorem 1 can be used to determine the centers of purity in torsion groups. The following result shows that the centers of purity in arbitrary groups can also be characterized.

THEOREM 2. A subgroup $H$ of an abelian group $G$ is a center of purity in $G$ if and only if the following two conditions are satisfied:

(i) the torsion subgroup $H_{t}$ of $H$ is a center of purity in the torsion subgroup $G_{t}$ of $G$;

(ii) either $G / H$ is a torsion group, or else, for all primes $p$,

$$
H[p] \subseteq \bigcap_{n=0}^{\infty} p^{n} G
$$

The problem of characterizing centers of purity in $p$-groups was first posed by J. M. Irwin in [2]. Irwin showed that any subgroup of a $p$-group $G$ which is maximal disjoint from $G^{1}$ is pure in $G$. In [3], Irwin and Walker extended this result to arbitrary abelian groups. They also showed that if $G$ is a torsion group and $H$ is a subgroup

Received May 16, 1962, and in revised form July 12, 1962. This paper was written while the author was a Senior Postdoctoral Fellow of the National Science Foundation at the Iniversity of California, Berkeley.

1 In this issue. 
of $G^{1}$, then $H$ is a center of purity in G. Charles pointed out that the proof given in [1] of Erdélyi's theorem (see p. 81) shows that the subgroups $p G, p^{2} G, p^{3} G, \cdots$ of a $p$-group $G$ are centers of purity. Khabbaz (in [4]) showed how the proof of Erdélyi's theorem could be modified to obtain a short proof of Irwin and Walker's result. Finally, Reid established the general sufficient condition 2.1 of [5] for a subgroup $H$ of an arbitrary group $G$ to be a center of purity. It was Reid who introduced the term "center of purity." In the lemma below, we show that Reid's condition is necessary as well as sufficient for $H$ to be a center of purity in a p-group $G$. This lemma is then used to prove Theorem 1, from which Theorem 2 follows easily. The author is indebted to Professor Reid for sending him a pre-print of the paper [5]. It was the reading of this paper which inspired the present work.

The notation and terminology of [5] will be used in this paper. In addition, we let $O(x)$ denote the order of the element $x$.

LEMMA. Let $G$ be a p-group, and suppose that $H$ is a subgroup of $G$. Then there is a subgroup $M$ of $G$ such that $M$ is maximal with respect to disjointness from $H$, and $M$ is not pure in $G$, if and only if the following conditon is satisfied.

(*) There exists $h \in H$ and $m \in G$ such that

(i) $O(m)>O(h)=p$;

(ii) $h_{p}(m)=h_{p}(h)<h_{p}(m+h)$;

(iii) $\{m\} \cap H=0$.

Proof. Suppose that $M$ is a subgroup of $G$ which is maximal disjoint from $H$ and not pure in $G$. Using the fact that two subgroups of a $p$-group are disjoint if and only if their $p$-layers are disjoint, it is easy to see that $M$ is maximal disjoint from $H[p]$. Therefore $(*)$ is satisfied by Theorem 2.1 of [5]. (It is clear from the proof of 2.1 that $p m \neq 0$, so that $O(m)>O(h)$.)

Assume conversely that the condition $(*)$ is satisfied. Let $r$ be a natural number such that $h_{p}(m)<r \leqq h_{p}(m+h)$. Define $P_{r}=$ $p^{r} G \cap G[p]$. Let $O(m)=p^{j}$, where, by $(i), j>1$. Then by (i), $n=$ $p^{j-1} m=p^{j-1}(m+h)$ has height $\geqq r+1$. Thus, $n \in P_{r}$. However, by (iii), $n \notin H[p]$. Consequently, there is a vector space decomposition

$$
P_{r}=S \oplus\left(P_{r} \cap H[p]\right), \quad n \in S .
$$

By (i) and (ii), $h \in H[p]$ and $h \notin P_{r} \cap H[p]$. Therefore, there is a decomposition

$$
H[p]=T \oplus\left(P_{r} \cap H[p]\right), \quad h \in T .
$$


Clearly,

$$
P_{r}+H[p]=S \oplus T \oplus\left(P_{r} \cap H[p]\right) .
$$

Finally, choose a decomposition

$$
G[p]=R \oplus\left(P_{r}+H[p]\right) .
$$

Define

$$
M_{0}=R \oplus S
$$

Then we have

$$
G[p]=M_{0} \oplus H[p], \quad \text { with } n \in M_{0} .
$$

Let $\pi$ be the projection mapping determined by this decomposition:

$$
\pi: G[p] \rightarrow H[p] \text {. }
$$

Note that by the construction, $\pi\left(P_{r}\right)=P_{r} \cap H[p]$. Define

$$
K=\left\{M_{0}, m\right\} \text {. }
$$

It is easy to see that since $p^{j-1} m=n \in M_{0}$, the $p$-layer of $K$ is $M_{0}$. Thus, $K[p] \cap H[p]=M_{0} \cap H[p]=0$, and therefore $K \cap H=0$. Let $M$ be maximal containing $K$ and disjoint from $H$. The proof of the lemma is completed by showing that $h_{p}^{M}(p m) \leqq r$. Indeed, this will imply that $M$ is not pure, because

$$
h_{p}(p m)=h_{p}(p(m+h)) \geqq h_{p}(m+h)+1 \geqq r+1 .
$$

Suppose that $h_{p}^{M}(p m) \geqq r+1$. Then $z \in M$ exists satisfying

$$
p^{r+1} z=p m \text {. }
$$

Consequently,

$$
u=p^{r} z-m \in M \cap G[p]=M \cap\left(M_{0}+H[p]\right)=M_{0}+(M \cap H[p])=M_{0} \text {. }
$$

Since $h_{p}(m+h) \geqq r$, we can write

$$
m+h=p^{r} y
$$

for some $y \in G$. Thus,

$$
p^{r}(y-z)=h-u \in G[p] \cap p^{r} G=P_{r},
$$

and therefore since $u \in M_{0}$,

$$
h=\pi(h-u) \in \pi\left(P_{r}\right)=P_{r} \cap H[p] \subseteq P_{r} .
$$

However, $h_{p}(h)<r$ by the choice of $r$. This contradiction shows that $h_{p}^{M}(p m)>r$ is impossible, so that the proof of the lemma is complete. 
We can now prove Theorem 1. Suppose that $P_{k} \supseteqq H[p] \supseteqq P_{k+2}$. If $k=\infty$, there cannot be any $h \in H[p]$ satisfying condition (ii) of the lemma. Suppose therefore that $k$ is finite. Assume that $h \in H$ and $m \in G$ exist satisfying conditions (i), (ii) and (iii) of $\left(^{*}\right)$ in the lemma. Let $h_{p}(h)=j$. Then $k \leqq j<h_{p}(m+h) \leqq \infty$. Let $O(m)=p^{f}$, where $f \geqq 2$ by (i). Write $x=m+h$. Then $h_{p}(x) \geqq k+1$. Consequently, $h_{p}\left(p^{f-1} x\right) \geqq k+2$. Therefore, $p^{f-1} m \in P_{k+2} \subseteq H[p]$. This is contrary to (iii). It follows that $H$ is a center of purity in $G$. Conversely, suppose that $P_{k} \supseteqq H[p] \supseteqq P_{k+2}$ is not satisfied for any $k$. Then in particular, $H[p] \nsubseteq P_{\infty}$. Since $P_{\infty}=\bigcap_{k<\omega} P_{k}$, it follows that $H[p] \nsubseteq P_{j}$ for some finite $j$. Let $k \geqq 0$ be the largest natural number such that $H[p] \subseteq P_{k}$. The maximality of $k$ and the fact that $P_{k} \supseteqq$ $H[p] \supseteqq P_{k+2}$ is false implies that

$$
H[p] \nsubseteq P_{k+1}, \quad H[p] \cong P_{k}, \quad \text { and } \quad P_{k+2} \nsubseteq H[p] .
$$

Therefore, there is an element $h \in H[p]$ such that $h_{p}(h)=k$, and there exists $u \in P_{k+2}$ such that $u \notin H[p]$. Let $u=p v$, where $v \in G$ and $h_{p}(v) \geqq k+1$. Define $m=v-h$. Then $O(m)=p^{2}>p=O(h), h_{p}(m)=$ $k, \quad h_{p}(m+h)=h_{p}(v) \geqq k+1$, and $\{m\} \cap H=0$, since $p m=p v=$ $u \notin H[p]$. It follows from the lemma that $H$ is not a center of purity in $G$. The proof of Theorem 1 is therefore complete.

Theorem 2 is obtained with the help of Theorem 1, by refiningthe proof of Lemmas 3.5 and 3.7 in [5]. Suppose that $G / H$ is a. torsion group, and $H_{t}$ is a center of purity in $G_{t}$. If $M$ is maximal disjoint from $H$, then $M \subseteq G_{t}$, and a short calculation shows that $M$ is maximal disjoint from $H_{t}$ in $G_{t}$. Therefore $M$ is pure in $G_{t}$, and hence also in $G$. Next, suppose that $H[p] \subseteq \bigcap_{n=0}^{\infty} p^{n} G$ for all primes $p$. If $H$ is not a center of purity in $G$, then by Theorem 2.1 in [5], there exists $h \in H_{p}$ such that $h_{p}(h)<\infty$. Let $O(h)=p^{r}$. Using the same argument that was given in the last paragraph of the proof of 2.1 in [5], we can show that $h_{p}\left(p^{r-1} h\right)<\infty$. This contradiction proves that $H$ must be a center of purity. Suppose conversely that $H$ is a center of purity in $G$. It is a routine exercise to show that $H_{t}$ is a. center of purity in $G_{t}$. Assume that $G / H$.is not a torsion group and for some prime $p, H[p] \nsubseteq \bigcap_{n=0}^{\infty} p^{n} G$. Let $k$ be the largest integer such that $p^{k} G \supseteqq H[p]$. Then by Theorem 1

$$
p^{k} G \cap G[p] \supseteqq H[p] \supseteqq p^{k+2} G \cap G[p], \quad p^{k+1} G \cap G[p] \nsupseteq H[p] .
$$

Let $t \in H[p]$ satisfy $h_{p}(t)=k$. Since $G / H$ is not a torsion group, an element $x \in G$ exists satisfying $O(x)=\infty$ and $\{x\} \cap H=0$. Consequently $\left\{p^{k+2} x+t\right\} \cap H=0$. Let $M$ be maximal disjoint from $H$, with $p^{k+2} x+t \in M$. Then $p^{k+3} x=p\left(p^{k+2} x+t\right) \in M$. Since $H$ is a center of purity, $M$ is pure. Consequently, $m \in M$ exists satisfying $p^{k+3} m=$ 
$p^{k+3} x$. Thus, $p^{k+2}(x-m) \in p^{k+2} G \cap G[p] \subseteq H[p]$. Therefore,

$$
p^{k+2} x+t-p^{k+2} m=p^{k+2}(x-m)+t \in H \cap M=0,
$$

so that $h_{p}(t) \geqq k+2$. However, $h_{p}(t)=k$ by choice. The contradiction shows that the condition (ii) must hold.

\section{REFERENCES}

1. L. Fuchs, Abelian groups, Budapest, 1958.

2. J. M. Irwin, High subgroups of Abelian torsion groups, Pacific J. Math., 11 (1961), $1375-1384$.

3. J. M. Irwin and E. A. Walker, On N-high subgroups of abelian groups, Pacific J. Math., 11 (1961), 1363-1374.

4. Samir Khabbaz, On a theorem of Charles and Erdélyi, Bull. Soc. Math. France, 89 (1961), 103-104.

5. J. D. Reid, On subgroups of an abelian group maximal disjoint from a given subgroup, Pacific J. Math., 13 (1963), to appear.

University of Washington, Seattle 



\section{PACIFIC JOURNAL OF MATHEMATICS}

\section{EDITORS}

\section{RalPh S. Phillips}

Stanford University

Stanford, California

M. G. Arsove

University of Washington

Seattle 5, Washington
J. Dugundji

University of Southern California Los Angeles 7, California

Lowell J. Paige

University of California

Los Angeles 24, California

\section{ASSOCIATE EDITORS}
E. F. BECKENBACH
D. DERRY
H. L. ROYDEN
E. G. STRAUS
T. M. CHERRY
M. OHTSUKA
E. SPANIER
F. WOLF

\section{SUPPORTING INSTITUTIONS}

\author{
UNIVERSITY OF BRITISH COLUMBIA \\ CALIFORNIA INSTITUTE OF TECHNOLOGY \\ UNIVERSITY OF CALIFORNIA \\ MONTANA STATE UNIVERSITY \\ UNIVERSITY OF NEVADA \\ NEW MEXICO STATE UNIVERSITY \\ OREGON STATE UNIVERSITY \\ UNIVERSITY OF OREGON \\ OSAKA UNIVERSITY \\ UNIVERSITY OF SOUTHERN CALIFORNIA
}

STANFORD UNIVERSITY

UNIVERSITY OF TOKYO

UNIVERSITY OF UTAH

WASHINGTON STATE UNIVERSITY

UNIVERSITY OF WASHINGTON

AMERICAN MATHEMATICAL SOCIETY

CALIFORNIA RESEARCH CORPORATION SPACE TECHNOLOGY LABORATORIES

NAVAL ORDNANCE TEST STATION

Printed in Japan by International Academic Printing Co., Ltd., Tokyo Japan 


\section{Pacific Journal of Mathematics}

\section{Vol. 13, No. 1 \\ March, 1963}

Frantz Woodrow Ashley, Jr., A cone of super-(L) functions............. 1

Earl Robert Berkson, Some metrics on the subspaces of a Banach space....

Felix Earl Browder and Walter Strauss, Scattering for non-linear wave

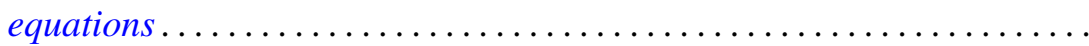

Edmond Darrell Cashwell and C. J. Everett, Formal power series ..........

Frank Sydney Cater, Continuous linear functionals on certain topological

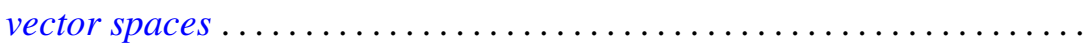

John Douglas Dixon, General group extensions ....................

Robert Pertsch Gilbert, On harmonic functions of four variables with

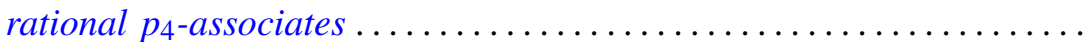

Irving Leonard Glicksberg, On convex hulls of translates ..............

Simon Hellerstein, On a class of meromorphic functions with deficient zeros

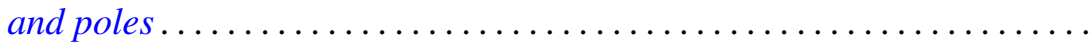

Donald William Kahn, Secondary cohomology operations which extend the

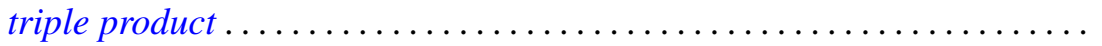

G. K. Leaf, A spectral theory for a class of linear operators .............

R. Sherman Lehman, Algebraic properties of the composition of solutions of partial differential equations ........................... 157

Joseph Lehner, On the generation of discontinuous groups ............. 169

S. P. Lloyd, On certain projections in spaces of continuous functions ...... 171 Fumi-Yuki Maeda, Generalized spectral operators on locally convex spaces ..................................

Donald Vern Meyer, $E^{3}$ modulo a 3-cell

William H. Mills, An application of linear programming to permutation groups.

Richard Scott Pierce, Centers of purity in abelian groups

Christian Pommerenke, On meromorphic starlike functions ...

Zalman Rubinstein, Analytic methods in the study of zeros of

polynomials...

B. N. Sahney, On the Nörlund summability of Fourier series

Tôru Saitô, Regular elements in an ordered semigroup . .

Lee Meyers Sonneborn, Level sets on spheres...........

Charles Andrew Swanson, Asymptotic estimates for limit point

problems .

Lucien Waelbroeck, On the analytic spectrum of Arens . .

Alvin (Murray) White, Singularities of a harmonic function of three

variables given by its series development .............

Kōichi Yamamoto, Decomposition fields of difference sets ...

Chung-Tao Yang, On the action of $\mathrm{SO}(3)$ on a cohomology manifold... 